\title{
Investigative determination of rod matrix in pulsating high gradient magnetic separation of ultrafine hematite tailings
}

\author{
Huifen Zhang, a and Zhihua Qian ${ }^{2, \text { b }}$ \\ ${ }^{1}$ Department of Land Resource Engineering, Yunnan Tin College of Vocation and Technology, Gejiu \\ 661000, China; \\ ${ }^{2}$ SLon Magnetic Separator Ltd., Ganzhou 341000, China; \\ a zank800@126.com, ${ }^{\mathrm{b}}$ 16684067@qq.com
}

Keywords: Pulsating high gradient magnetic separation; Rod matrix; Ultrafine hematite tailings Abstract: A mass of ultrafine weakly magnetic iron minerals are produced during the processing of iron ores such as hematite and limonite, and in the most cases these fine minerals are recovered at a very low efficiency, especially for the magnetic particles below $20 \mu \mathrm{m}$. Pulsating high gradient magnetic separation (HGMS) of rod matrix is comparatively investigated for the processing of an ultrafine hematite tailings (around 65\% below $20 \mu \mathrm{m}$ ), to determine the optimum rod matrix for the recovery of ultrafine hematite minerals from the tailings. The results of investigation indicate the finest $1 \mathrm{~mm}$ rod matrix achieved the most effective processing of the tailings assaying $28.99 \% \mathrm{Fe}$, produced a concentrate assaying $46.85 \%$ Fe with $59.39 \%$ recovery at a low magnetic induction of 0.5 $\mathrm{T}$, and these are much superior to those of the $2 \mathrm{~mm}$ and $3 \mathrm{~mm}$ rod matrixes.

\section{Introduction}

A mass of ultrafine weakly magnetic iron minerals are produced during the processing of iron ores such as hematite and limonite (Zheng et al., 2015), and in the most cases these fine minerals are concentrated at a very low efficiency, especially for the magnetic particles smaller than $20 \mu \mathrm{m}$. It has even been estimated that 120 to $130 \mathrm{Mt}$ iron tailings containing more than $14 \mathrm{Mt}$ iron values is discarded as tailings per year in China, due to insufficient technology available to handle such tailings (Chen et al., 2009). In practice, pulsating high gradient magnetic separation (HGMS) presents the most effective method for the concentration of fine hematite (Zeng and Xiong, 2003).

In the present investigation, pulsating HGMS of rod matrix is comparatively investigated for the processing of an ultrafine hematite tailings (around $65 \%$ below $20 \mu \mathrm{m}$ ), to determine the optimum rod matrix for the recovery of ultrafine hematite minerals from the tailings.

\section{Materials and methods}

\section{Characteristics of material}

A hematite tailings assaying $28.99 \%$ Fe was used in the investigation and dominant gangues in the material are quartz and muscovite, etc. As shown in Table 1, particles are ultrafinely distributed in the material, with $78.43 \%$ of the material distributed in the ultrafine range below $30 \mu \mathrm{m}$; this fraction is rich in iron, accounting for $86.16 \%$ of the total iron in the material. It is noted that $47.48 \%$ of the material is in the extremely ultrafine range below $10 \mu \mathrm{m}$, accounting for $48.28 \%$ of the total iron. 
Table 1 Size analysis of material.

\begin{tabular}{cccc}
\hline Size range $(\mu \mathrm{m})$ & Weight $(\%)$ & Grade $(\% \mathrm{Fe})$ & Iron distribution $(\%)$ \\
\hline+74 & 4.53 & 17.28 & 2.70 \\
$-74+37$ & 11.90 & 18.62 & 7.64 \\
$-37+30$ & 5.14 & 19.75 & 3.50 \\
$-30+20$ & 13.71 & 34.53 & 16.33 \\
$-20+10$ & 17.24 & 36.23 & 21.55 \\
-10 & 47.48 & 29.48 & 48.28 \\
\hline Total & 100.0 & 28.99 & 100.0 \\
\hline
\end{tabular}

\section{Cyclic pilot-scale Pulsating HGMS separator}

A SLon-100 cyclic pilot-scale pulsating HGMS separator (Fig.1) was used to concentrate the hematite tailings. In the investigation, 1, 2 and $3 \mathrm{~mm}$ diameter round bars made of magnetic stainless steel are used as magnetic matrix in the separator, and they are varied in the configuration.

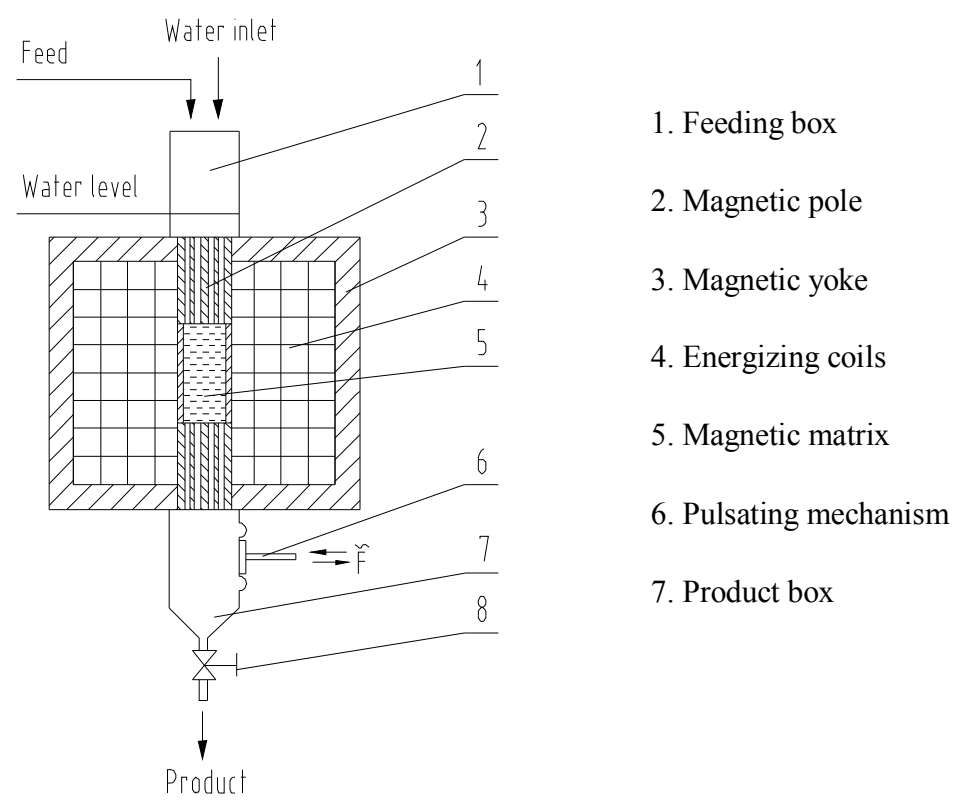

Fig. 1. SLon-100 cyclic pilot-scale pulsating HGMS separator.

While the separator is being operated, a direct current flows through the energizing coils and a magnetic field is built up in the separating zone. Firstly, the separating zone is filled with flowing water so that the pulsating energy is transmitted to the separating zone, in which the level of water and its flowrate are adjustable by the valve below the pulsating mechanism. Then, the slurry is fed into the matrix in the separating zone through the feeding box. Magnetic particles are attracted from slurry onto the surface of matrix, while non-magnetic particles pass through the matrix and go out through the product box to become tailings under the combined actions of slurry pulsation, gravity and hydrodynamic drag. The pulsating mechanism drives the slurry in the separating zone up and down, keeping particles in the matrix in a loose state so that magnetic particles are more easily captured by the matrix and non-magnetic particles are more easily dragged out through the matrix. 
The separator is fed periodically. When a batch of feed is finished, the energizing current is switched off and magnetic particles in the matrix are washed out to get a magnetic product. A more detailed description on the pulsating HGMS is recently reported by Chen et al. (2014).

The material of $200 \mathrm{~g}$ was fully mixed in a stir beaker at a $10 \%$ solid concentration and then fed to the separator within 10-15 seconds. For all the separations, pulsating frequency is controlled at a gentle level of $225 \mathrm{r} / \mathrm{min}$, while the magnetic induction is varied.

\section{Results and discussion}

\section{Effect of magnetic induction on pulsating HGMS performance of $1 \mathrm{~mm}$ rod matrix}

The finest $1 \mathrm{~mm}$ rod matrix is firstly used in the separator to process the ultrafine tailings, due to the ultrafine distribution characteristics of the material. It can be seen from Fig.2, it achieved an effective recovery for the fine magnetic hematite from tailings; as the magnetic induction increased, the iron recovery rapidly increased and then approached the maximum around $50 \%$, while the concentrate grade decreased with decreasing magnetic induction and this is due to the increasing magnetic capture to magnetic particles by the matrix (Chen et al., 2009).

It is interesting to note that this finest $1 \mathrm{~mm}$ rod matrix is capable of achieving a high separation performance at a magnetic induction as low as $0.5 \mathrm{~T}$.
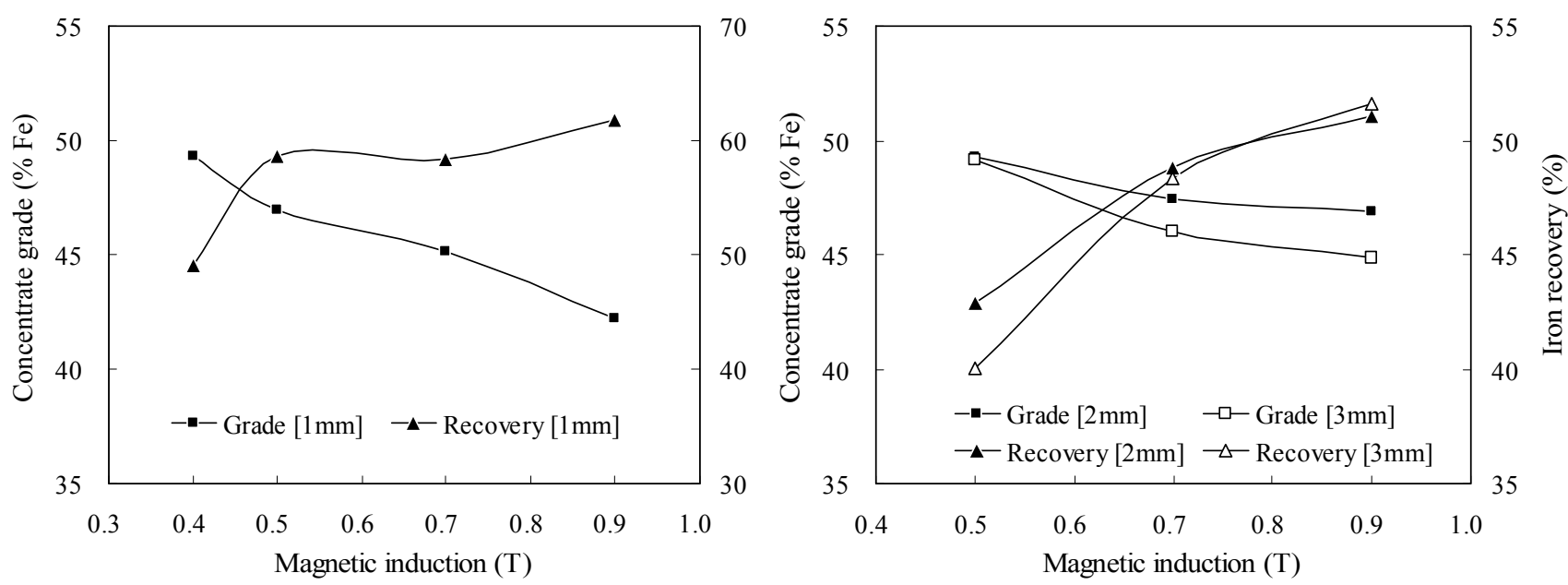

Fig.2. Effect of magnetic induction on pulsating HGMS performance of rod matrix for $1 \mathrm{~mm}(\mathrm{left})$ and 2 and $3 \mathrm{~mm}$ (right) rod matrixes.

\section{Effect of magnetic induction on pulsating HGMS performance of 2 and $3 \mathrm{~mm}$ rod matrixes}

The material is further processed with coarser 2 and $3 \mathrm{~mm}$ rod matrix with the cyclic pilot-scale pulsating HGMS separator, with results as shown in Fig.2. The two matrixes followed the similar trend; as the magnetic induction increased, the concentrate grade decreased with reduction in the iron recovery. It is clear that both the two matrix achieved inferior separation performance to that of the finest 1 rod matrix, even at a much higher magnetic induction reaching as high as $0.9 \mathrm{~T}$.

Combining the separation results of 1,2 and $3 \mathrm{~mm}$ rod matrixes in Fig.2, the finest $1 \mathrm{~mm}$ rod matrix is well suited for the recovery of ultrafine hematite minerals from the tailings, producing a much superior separation performance at a low electric consumption cost. 


\section{Performance comparison between 1, 2 and $3 \mathrm{~mm}$ rod matrix}

The above separation tests are comparatively repeated at the definitely same operation conditions, i.e., at a pulsating frequency of $225 \mathrm{r} / \mathrm{min}$, at a feed weight of $200 \mathrm{~g}$ and at a feed solid concentration of $10 \%$, except for the magnetic induction. It can be seen from Table 2, while the $1 \mathrm{~mm}$ rod matrix produced a similar concentrate grade around $49 \%$ Fe to the 2 and $3 \mathrm{~mm}$ rod matrixes, it achieved a much higher iron recovery approaching 50\% at a much lower magnetic induction of $0.4 \mathrm{~T}$. Under the optimum conditions (at a magnetic induction of $0.5 \mathrm{~T}$ ), this finest rod matrix produced a concentrate grade assaying $46.85 \% \mathrm{Fe}$ at an iron recovery as high as $59.39 \%$, with the tailings grade assaying $18.61 \% \mathrm{Fe}$, much lower than those (higher than $22 \% \mathrm{Fe}$ ) of the 2 and $3 \mathrm{~mm}$ rod matrixes.

Table 2 Performance comparison between 1, 2 and $3 \mathrm{~mm}$ rod matrixes.

\begin{tabular}{cccccc}
\hline \multirow{2}{*}{ Rod matrix } & \multicolumn{3}{c}{ Concentrate } & Tailings grade & $\begin{array}{c}\text { Magnetic } \\
\text { induction }(\mathrm{T})\end{array}$ \\
\cline { 2 - 4 } & Weight $(\%)$ & Grade $(\% \mathrm{Fe})$ & Iron recovery $(\%)$ & 20.58 & 0.4 \\
\multirow{2}{*}{$1 \mathrm{~mm}$} & 29.27 & 49.32 & 49.80 & 18.61 & 0.5 \\
$2 \mathrm{~mm}$ & 36.75 & 46.85 & 59.39 & 22.06 & 0.7 \\
$3 \mathrm{~mm}$ & 25.47 & 49.26 & 43.28 & 22.95 & 0.9 \\
\hline
\end{tabular}

\section{Conclusions}

The finest $1 \mathrm{~mm}$ rod matrix is well suited for the recovery of hematite minerals from the ultrafine tailings at a low electric consumption cost. At a low magnetic induction of $0.5 \mathrm{~T}$, it produced a concentrate grade assaying $46.85 \% \mathrm{Fe}$ with recovery reaching as high as $59.39 \%$ from the tailings assaying $28.99 \% \mathrm{Fe}$, with its tailings grade as low as $18.61 \% \mathrm{Fe}$; such a separation performance is much superior to the 2 and $3 \mathrm{~mm}$ rod matrixes.

\section{Acknowledgements}

This work was financially supported by the Educational Key Program of Yunnan Province of China (Grant No. 2014C009Z).

\section{References}

[1] X. Y. Zheng, Y. H. Wang, D. F. Lu. Study on capture radius and efficiency of fine weakly magnetic minerals in high gradient magnetic field. Minerals Engineering. 2015, 74: 79 85.

[2] L. Z. Chen, D. H. Xiong, H. C. Huang, Pulsating high-gradient magnetic separation of fine hematite from tailings. Minerals \& Metallurgical Processing. 2009, 26 (3): 163 168.

[3] W. Q. Zeng, D. H. Xiong. The lastest application of Slon vertical ring and pulsating high-gradient magnetic separator. Minerals Engineering. 2003, 16: 563 565.

[4] L. Z. Chen, L. Ding, H. F. Zhang, J. X. Huang. Slice matrix analysis for combinatorial optimization of rod matrix in PHGMS. Minerals Engineering. 2014, 58: 104 107. 
